(Aus dem Psychologischen Institut der Universität Tiflis in Georgien.)

\title{
Ein experimenteller Beitrag zum Problem der psychologischen Grundlagen der Namengebung.
}

Von

D. Usnadze.

Zwei charakteristische Momente sind es, die im Prozesse des Werdeganges des Lautkomplexes zum Wort als grundlegend anzusprechen sind: das Moment der Bedeutungsverleihung im allgemeinen und das Moment der bestimmten Bedeutungsverleihung, in den nämlich ein Lautkomplex als Träger einer fest umgrenzten Bedeutung, als Zeichen, als Name erlebt wird. In beiden Fällen ist der Lautkomplex zum Wort geworden, aber indem er auf der ersten Stufe bloß als Zeichen, als Ausdruck einer strukturellen Gesamtlage des Bewußtseins, eines Affektes oder eines Wunsches hervortritt [z. B. „Mutter" ist für das Kind nicht als Bezeichnung eines bestimmten Wesens anzusehen, sondern allein als der Ausdruck eines im gegebenen Moment bestimmten Wunsches, den es gern befriedigen möchte $\left.{ }^{1}\right]$ ), wird er auf der zweiten Stufe zur Bezeichnung eines intellektuell erfaßten, objektiven, gegenständlichen Inhaltes verwendet. Wenn der Lautkomplex also auf der ersten Stufe bloß als Ausdruck einer Gesamtlage des subjektiven Bewußtseins benutzt würde, so ist er jetzt zum Träger einer neuen Funktion, und zwar der Funktion der Benennung geworden.

Es ist klar, daß im Werdegang der Lautkomplexe zur Sprache dieses zweite Moment als entscheidend zu betrachten ist. Erst hier werden die Lautkomplexe abgesondert, in ihrer. Individualität erfaßt, als selbständige Träger bestimmter Inhalte bewußt. Hier werden wir zuerst der endgültigen Begegnung des Gedankens mit dem Lautkomplex, also der Geburt des eigentlichen Wortes gewahr, und wir müssen anerkennen, daß wir hier mit einem Anfangsdatum der Geschichte der wirklichen Sprache zu tun haben.

Kein Wunder, daß wir ein großes Interesse der Erfassung dieses gleichsam grundlegenden Momentes entgegenbringen: Wie ist denn eigentlich ein Lautkomplex zum Wort, und zwar zum Namen geworden?

1) W. Stern, Psychologie der frühen Kindheit. 3. Aufl. Leipzig 1923. - K. Bühler, Die geistige Entwicklung des Kindes. 3. Aufl. 1922, S. 222. 
Was hat demselben die Namensfunktion verliehen? Ohne Zweifel, diese Frage richtet unsere Aufmerksamkeit auf die Erleuchtung der verborgenen Bedingungen der Entstehung der Sprache, der Erfassung der ersten Schritte ihrer Entwicklung. Nun hat Paul mit Recht darauf hingewiesen, daß die Erforschung des Ursprungs und der Entwicklung der Sprachformen nur durch die Vergegenwärtigung dessen möglich ist, was zwar in den Bestandteilen der Sprache nicht enthalten ist, den Sprechenden aber sowohl wie den Hörenden jeden Augenblick vorschwebt ${ }^{1}$ ). Er hat dabei wohl das psychologische Moment gemeint, und es ist in der Tat unbestreitbar, daß das Problem ohne gründliche Erforschung der psychologischen Seite der Entwicklung der Sprache nicht zu lösen ist. Eben deswegen ist es besonders zu bedauern, daß die moderne Psychologie sich wenig mit der Frage beschäftigt hat. Nun war es von ihr auch nicht anders zu erwarten, solange sie die Probleme der komplizierten psychischen Phänomene bloß mit Hilfe der historischen, genetischen und vergleichenden Methoden zu lösen trachtete. Seitdem aber die psychologische Forschung sich anschickte, auch diesen Problemkreis experimentell $\mathrm{zu}$ bewältigen, eröffnete sich für das Problem der sprachlichen „Urschöpfung" eine hoffnungsvolle Perspektive.

$S$. Fischer hat neulich das Problem vom ,Entstehen und Verstehen der Namen": experimentell zu lösen in Angriff genommen, und einige wichtige Resultate auch für unser Problem erschlossen ${ }^{2}$ ). Im weiteren Forschen könnte man den Grundgedanken seiner Methode behalten, indem man dieselbe der abweichenden Fragestellungen gemäß umänderte. So ist es auch in der vorliegenden Untersuchung geschehen. Bevor wir aber zu derselben übergehen, ist es notwendig, auf die Frage einzugehen, ob es überhaupt berechtigt ist, das Problem der psychologischen Grundlagen der Namengebung experimentell zu behandeln. Sofern sich die Frage auf die Grundlagen der auf der Tatsache einer entwickelten Sprache fußenden Benennung bezieht, ist sie wenig bestreitbar. Sofern sie aber weitergeht und sich auf die ursprünglichen, $d . h$. primären Grundlagen der Namensgebungsfunktion richtet, erweckt die Fruchtbarkeit der Experimentalforschung einen viel berechtigteren Zweifel.

Die Hauptschwierigkeit scheint dabei nämlich die zu sein, daß man für die in Betracht kommenden Versuche nicht einmal geeignete Versuchspersonen zu finden glaubt. Denn das Wesen der ,Urschöpfung“ besteht darin, daß die Verbindung zwischen Lautkomplex und Sachvorstellungen ohne Vermittlung der Vorstellungen geschehen muß,

1) Paul, Prinzipien der Sprachgeschichte. 5. Aufl. 1920, S. 36.

$\left.{ }^{2}\right)$ S. Fischer, Entstehen und Verstehen der Namen. Arch. f. d. ges. Psychol. 42, 339 . 
die irgendwie schon sprachlich benannt worden sind ${ }^{1}$ ). Der moderne Mensch aber atmet doch gleichsam nur die Atmosphäre der entwickelten Sprache; und wie könnte es möglich sein, daß er die Verknüpfung zwischen Lautkomplex und Sachvorstellung herstelle, ohne dabei auf die Vermittlung irgendeiner schon benannten Vorstellung zurïckzugreifen!

Dieser Einwand wird aber durch die Vergegenwärtigung der Tatsachen entkräftet, auf welche zum Teil schon Paul hingewiesen hat:

1. Es ist absolut nicht zu leugnen, daß die Schöpfung des neuen sprachlichen Materials auch in den Kultursprachen vor sich geht; sie ist bis heute nicht eingestellt und wird 'wahrscheinlich nie ganz eingestellt. Diejenigen Prozesse, die der sprachlichen Urschöpfung zugrunde lagen, haben also nie aufgehört zu wirken, und es ist klar, daß sie auch der modernen sprachlichen Neuschöpfungen zugrundegelegt sein müssen.

2. Der moderne Mensch steht nicht selten vor dem Problem der Namengebung: es werden neue Phänomene, neue Tatsachen und neue Begriffe entdeckt, die durch den sprachlichen Ausdruck noch nicht fixiert sind. Es entsteht die Aufgabe, dieselben zu benennen. Weil der Gegenstand, nach dessen Namen gesucht wird, evtl. außerhalb des Kreises der bekannten Dinge liegt, müssen die gewöhnlichen Worte als nicht zutreffend für seinen Ausdruck angesehen werden; sonst würden die unpassenden Assoziationen hervorgerufen, die auf die Vorstellung des neuen Inhalts assimilierend und folglich auch verwirrend einwirken würden. Es ist zu vermuten, daß, wenn überhaupt, so erst hier die elementaren Prozesse der Namengebung in Tätigkeit versetzt sein müssen.

Diese beiden Tatsachen genügen, erwähnt zu werden, um den oben hervorgehobenen Einwand gegen die Möglichkeit der Experimentalforschung innerhalb des uns hier interessierenden Problemreises wesentlich zu entkräften. Es ist freilich nicht zu übersehen, daß der moderne Mensch auf der Tatsache der entwickelten Sprache fußt. In dieser Beziehung ist er allerdings vom "Urschöpfungssubjekt" verschieden. Aber sobald wir mit dem Problem der Namengebung zu tun haben, müssen wir dessen eingedenk sein, $\mathrm{da} ß$ es sich hier um die Lautsprache handelt. Dieser aber ist bekanntlich eine ziemlich lange Vorgeschichte der Sprachentwicklung vorangegangen (die sog. Geberdensprache, die Sprache der Affekte und Wünsche u. ä.). Ein prinzipieller und absoluter Unterschied von dem heutigen Menschen ist also hier nicht zu konstatieren. Natürlich wird dieser nicht imstande sein, die Tatsache loszuwerden, daß er eben ein sprechendes Wesen ist, und er wird im Prozesse der Namengebung immer die Gelegenheit zu benutzen suchen,

1) Paul, op. cit. S. 176. 
auf den vorhandenen und ihm geläufigen Sprachschatz zurückzugreifen. Wenn es sich aber trotzdem herausstellen wird, daß der Prozeß der Namengebung auch Grundlagen aufweist, die nicht auf dem Sprachfaktum beruhen, und es nicht voraussetzen, so werden wir berechtigt sein, zu schließen, daß es eben die Grundlagen sind, die bei der sog. Urschöpfung besonders wirksam sein mußten.

Wir haben also vor allem die Faktoren zu ermitteln, die beim modernen Menschen im Prozesse der Namengebung in Wirksamkeit treten. Aus den Ergebnissen wäre dann auf primäre Faktoren zu schließen, die der Urschöpfung zugrunde lagen.

\section{Versuchsanordnung.}

Die Methode, der wir uns bei der Untersuchung bedienen, ist dje der systematischen Selbstbeobachtung. Es wurden 6 Zeichnungen hergestellt, die sinnlos gehalten werden mußten, damit sie möglichst keine Assoziationen mit bekannten Gegenständen hervorrufen könnten. Diese Zeichnungen wurden einzeln vor den Versuchspersonen je 5 Sek. lang exponiert, damit diese den passendsten Namen für sie ausfindig machen könnten. Es wurde ihnen aber nicht die Aufgabe gestellt, den Namen zu erfinden, sondern aus der überreichten Liste der vorher nach bestimmten Prinzipien zusammengestellten Lautkomplexe, die völlig unbekannt klangen, den für jede Figur passenden herauszufinden ${ }^{\mathbf{1}}$ ). Dann gaben die Versuchspersonen ihre Erlebnisse wieder, die sie im Prozesse des Versuchs hatten. An den Versuchen nahmen 10 Personen teil (im Lebensalter von 19-37 Jahren), deren Namen im weiteren mit Initialsilben bezeichnet werden, die Serien der Versuche aber mit römischen Ziffern.

\section{Die Ergebnisse.}

Das ganze Material, das uns zur Verfügung steht, läßt sich von 4 verschiedenen Fragestellungen aus analysieren:

1) Die Lautkomplexe wurden in der georgischen Schrift dargeboten und von den Versuchspersonen als georgische Laute ausgesprochen und aufgefaßt. Die georgische Sprache, deren linguistische Herkunft noch nicht endgültig geklärt ist, enthält eigenartige Laute, die sich durch die lateinische Schrift nicht ohne weiteres wiedergeben lassen, deshalb sehen wir uns genötigt, bei der Darstellung der Lautkomplexe durch die lateinische Schrift auf die Transkription zurückzugreifen und am besten auf diejenige, die in der Georgiologie am häufigsten gebraucht wird. Als solche betrachten wir die von Professor Marr eingeführte Transkription, bei der die am meisten eigenartig klingenden Laute folgenderweise wiedergegeben werden:

$\mathrm{j}=$ franz. $\mathrm{j} ; \mathrm{z}=$ franz. $\mathrm{z} ; \varphi=\mathrm{ph}($ griech. $\varphi) ; \dot{\mathrm{q}}=\operatorname{ch}($ hart $) ; \mathrm{q}=\mathrm{kh} ; \mathrm{t}=\mathrm{tsch}$ (empfat.); $d=$ ital. $\mathrm{g}$ (giorno); $\mathrm{d}=\mathrm{th} ; \breve{\mathrm{d}}=$ ital. zio; $\breve{\mathrm{g}}=(\mathrm{Ta}) \mathrm{g} ; \mathrm{k}=\mathrm{arab} . \mathrm{c}$; $\dot{\mathrm{D}}=\mathrm{z}$ (deutsch); $\mathfrak{d}=\mathrm{tsch} ; \check{\mathrm{s}}=\mathrm{sch} ; \dot{\mathrm{t}}=\mathrm{ts}$. Demnach wäre z. B. nagtaqi als nagtsachi zu lesen. 
1. Wie prägt sich die Wahmehmung eines Objektes aus, wenn dieselbe durch die Absicht der Namengebung geleitet wird?

2. Welches sind die Prozesse, die in Momente der Wahrnehmung der als Namen zu wählenden Lautkomplexe im Bewußtsein der Vpn. in Wirksamkeit treten?

3. Welche sind die Grundlagen, die den sinnlosen Lautkomplex zum Namen erheben?

4. Welche unter diesen Grundlagen sind als primäre und welche als abgeleitete und sekundäre anzusprechen?

a) Die Wahrnehmung des zu benennenden Objektes.

Bei der Wahrnehmung der Vorlage steht, wie gesagt, vor dem Bewußtsein der Versuchsperson folgende Aufgabe: Sie soll das dargebotene Objekt wahrnehmen und dann für dasselbe einen passenden Namen heraussuchen. Für einen sprechenden Menschen setzt jedes Wort unbedingt eine Bedeutung voraus. Das exponierte Objekt aber bedeutet zunächst so gut wie nichts, und eben diese Negation jeder Bedeutung muß die Bedeutung des herauszufindenden Wortes sein. Es liegt in der Natur der Sache, das die Vpn. sofort sich bemühen, einen Ausweg zu finden. Dabei verfahren sie einfach, und zwar, sie suchen aus dem komplexen Psychischen, das an der Hand der Vorlage hervortritt, irgendeine Bedeutung zu konstruieren oder herauszulesen. Und als der einfachste Weg empfiehlt sich dabei der der Angleichung des dargebotenen Objektes an etwas schon Bekanntes.

So z. B. sagt die Versuchsperson:

Sch. IV. Bei der Darbietung der Zeichnung kam sie mir völlig bedeutungslos vor. Ich verspürte aber das Bedürfnis, ihr irgendeinen Inhalt zu verleihen. Dio wellenförmigen Linien an der Zeichnung halfen mir, dieselben als Flammenzungen vorzustellen...

Oder die Versuchsperson:

Cha. III. Zunächst fiel mir njchts ein. Ich wollte aber unbedingt irgend etwas darin sehen. Schließlich gelang es mir ziemlich gut...

Am Schluß der Versuchsserie ließ ich die Versuchspersonen ihre Erlebnisse auch allgemein formulieren, und in bezug auf den Prozeß der Wahrnehmung des vorgelegten Objektes lautete ihre Beobachtung allgemein etwa folgendermaßen:

Bu. Beim Sehen der Zeichnungen war ich gewöhnlich bestrebt, dieselben mit irgendeinem bekannten Gegenstand zu vergleichen.

Diese allgemeine Tendenz der Versuchspersonen war so wirksam, daß einige derselben nach einigen Expositionen schon im voraus den Plan gefaßt hatten, in den dargebotenen Zeichnungen sofort etwas herauszufinden, das in irgendeiner Beziehung zu irgend etwas ihnen Bekanntem stehen könnte. Eine der Versuchspersonen macht sogar den Versuchsleiter darauf aufmerksam, daß sie sich eine derartige Hilfe schematisch für alle Versuche schon im voraus ausgearbeitet hatte: 
Cha. IV. Jetzt habe ich schon einen vorher bestimmten Plan, eine feste Determination. Ich bitte Sie darauf zu achten, denn vielleicht wird dadurch der Wert der Versuche herabgesetzt...

Der erste Eindruck ist derart, als ob die Versuchspersonen bei der Auffassung des neuen unbekannten Materials dasselbe mit dem sonst Bekannten zu vergleichen und von ihm zu unterscheiden strebe. Dieses Vergleichen und Unterscheiden kommt aber hier nur dann zustande, wenn sich die Versuchsperson nicht nur das Bemerken des dargebotenen Materials, sondern auch das Herauslesen irgendeiner Bedeutung aus demselben zum Ziel gesetzt hat.

Auf Grund unserer Versuche lassen sich 2 allgemeine Typen unterscheiden für das Finden der Bedeutung, die die Versuchspersonen in Anleitung der Benennungsintention im indifferenten Material sehen. Für den exsten Typus ist die direkte Beziehung auf das exponierte Material charakteristisch. Für ihn wird zum Träger der Bedeutung: entweder ein einzelner, irgendwie bekannter Gegenstand:

Tschv. II. Als ich die Zeichnung anschaute, stellte ich mir eine Fliege vor ...

2. Oder ein Bild, das die Versuchsperson irgendwo gesehen hat.

Tschi. VI. Die Zeichnung sagte mir zunächst nichts. Später aber tauchte im Bewußtsein ein Bild auf, das ich in einem Lehrbuch geschen habe, mit der Uberschrift: Die Seitenketten von Ehrlich... wird.

3. Oder endlich ein Buchstabe, der irgendeinem Teil der Zeichnung angeglichen

Me. IV. Die Zeichnung mutete mich an, wie das griechische (1).

Für diesen Typus ist also das Einzelne wichtig, und zwar dasjenige, das ihm in der Erfahrung irgendwann vorgekommen ist.

Der zweite Typus verhält sich einem Expositionsmaterial gegenüber freier und ungebundener. Irgendein Teil der Zeichnung wird an etwas einzelnes angegliedert; aber dieses einzelne regt sofort die Phantasie der Versuchsperson an, und sie stellen sofort Gesamtbilder her, in welche das einzelne sich als Bestandteil eingliedert und entweder klar, oder auch ganz dunkel apperzipiert wird. Die Vertreter dieses Typus lassen sich in 2 Untertypen klassifizieren: die einen entwickeln ihre phantastischen Bilder unter dem starken Einfluß der determinierenden Tendenz:

Tscho. II. Die Zeichnung stellt mich wieder auf Indien ein ${ }^{1}$ ). Ich sehe einen Indier mit einer Schlange in der Hand... er zieht sie heraus vom Busen .. sie umschlingt seinen Arm.

Die anderen aber scheinen ihre phantastischen Bilder in beliebiger Richtung entfalten zu können.

Scha. III. Die Zeichnung kam mir zunächst völlig sinnlos vor. Nachdem ich sie mir als Helm vorstellte, tauchten in meinem Bewußtsein die Bilder von alten Kriegern auf ... ich sehe die Schlacht, stelle mir wirklich die Laute vor, mit welchen die Kämpfer aufeinander losgehen.

1) Es wurde nämlich im Anschluß an S. Fischer die Zeichnung als Zeichen einer Geheimwissenschaft vorgeführt. 
Zwischen beiden Haupttypen ist noch ein Unterschied zu konstatieren: während die Vertreter des ersten Typus (wir möchten ihn als nüchternen Typus bezeichnen) sich durch die Kälte des Gefühls auszeichnen (das apperzeptive Material ist für sie emotionell völlig indifferent und nichtssagend), charakterisieren sich die Vertreter des zweiten, des phantasierenden Typus durch die Lebhaftigkeit des Gefühls: ihre phantastischen oder produktiv eingebildeten Bilderserien sind gewöhnlich (wenn nicht immer) durch einen lebhaften Gefühlston ausgezeichnet.

Wie, durch welche Seiten des Expositionsmaterials werden diese Einzelbilder oder Serien der Bilder bei beiden Typen hervorgerufen? Nach unseren Versuchen scheinen dabei 2 Wege in Betracht zu kommen:

1. Es wird irgendein Einzelteil der Zeichnung hervorgehoben und an etwas Bekanntes angeglichen:

Scha. II. Nach dem ersten Anblick schien die Assoziation mit etwas Bekanntem ganz zu versugen, denn das Ganze der Zeichnung sagte mir so gut wie nichts. Daher versuchte ich die Teile des Ganzen zu berücksichtigen und wenigstens künstlich irgendeine Ahnlichkeit mit etwas herzustellen. Nun fiel mir ein, daß ein Teil der Zeichnung wirklich an einen Fels erinnerte...

2. Es wird aber auch das Ganze der Zeichnung, ihre Vollgestalt in Betracht gezogen und die Assoziationen von hier aus ausgebreitet:

Scha. III. Zunächst fiel mir nichts ein. Auf mein Bemühen, die Zeichnung mit etwas Ähnlichem zu verknüpfen, wurde in mir die Vorstellung wach, die der Gestalt der Zeichnung paßte.

Damit sind aber noch lange nicht alle Fälle der Bedeutungsverleihung erschöpft. Es kommen Fälle vor, bei denen die Wahrnehmung des Expositionsmaterials eine ganz andere Richtung einschlägt, und zwar, trotz des intensiven Bemühens gelingt es den Versuchspersonen nicht, das vorgezeigte Objekt, hier die Zeichnung, mit irgendeinem bestimmten, bekannten objektiven Inhalt in Verbindung zu bringen. In solchen Fällen greift die Versuchsperson auf einen der folgenden 4 Wege:

1. Die Versuchsperson analysiert die angeschaute Figur und stellt die Relation ihrer Teile fest; dann hilft sie sich durch den Gedanken dieser Relation, die sie dann in der Wahrnehmung besonders klar apperzipiert.

$\mathrm{Bu}$. IV. Ich konnte nichts herausfinden. Doch habe ich bald bemerkt, daß die Zeichnung eigentlich aus zwei Teilen bestand. Anch ferner wurde ich von diesem Gedanken geleitet.

2. Einige Versuchspersonen beschränkten sich auf die allgemeine; manchmal ganz abstrakte Charakterisierung der Zeichnung.

Cha. V. Ich habe meine Aufmerksamkeit auf die Eckseite der Zeichnung gelenkt und wollte mir im Anschluß daran irgendeine bekannte Vorstellung hervorrufen. Das gelang mir jedoch nicht. Nun habe jch die Gestalt der Zeichnung fixiert, 
und jetzt kam mir der Gedanke, daß diese Zeichnung wirklich gut zum Zeichen einer Geheimschrift passen könnte ... Weiter ließ ich mich durch diesen Gedanken leiten...

3. Es gibt aber auch Versuchspersonen, welchen zum Repräsentanten der Bedeutung des Expositionsobjektes eine emotionelle Gesamtlage dient.

Tsi. IV. Konnte nichts herausfinden. Es blieb allein ein klarer Eindruck des Angenehmen, den die anmutige Gestalt der Figur hervorgerufen hat.

4. Es gibt noch einen Weg, der ganz besonders interessant zu sein scheint. Die Versuchsperson hat nämlich im Bewußtsein weder eine anschauliche Vorstellung als eine Bedeutung des Expositionsmaterials noch einen allgemeinen Gedanken über dasselbe, noch endlich einen emotionellen Eindruck; trotzdem aber geht etwas in ihr vor, und dies bezeichnet sie, nicht gerade sehr charakteristisch, als einen ,allgemeinen Eindruck". Was denn eigentlich dieser , allgemeine Eindruck“ ist, darauf wollen wir nicht eingehen; wir begnügen uns hier, die Tatsache allein zu konstatieren; z. B.:

Tsi. II. Die Zeichnung habe ich mit nichts in Verbindung gebracht. Aber ich bekam gleich einen allgemeinen, nicht näher zu bestimmenden Eindruck, und nun leitete mich bei der Namengebung dieser Eindruck. Ich wählte einen Komplex aus, der mir am geeignetsten erschien; warum, kann ich nicht sagen; ich fühle aber bestimmt, daß dieser Komplex wirklich am besten paßt.

\section{b) Die Wahrnehmung der Lautkomplexe.}

Was geschieht nun im Bewußtsein der Versuchspersonen, wenn diese die Lautkomplexe wahrnehmen, unter denen sie einen für die Zeichnung passenden Namen auszuwählen haben?

Die Versuchsperson nimmt die Lautkomplexe wahr in der Absicht, unter diesen den der vorgeführten Zeichnung zustehenden Namen auszuwählen; andererseits sucht sie doch eben unter der Intention der Namengebung nach der ,Bedeutung" des exponierten Objektes. Es ist klar, daß sie unter diesen Bedingungen die Lautkomplexe eben unter der Determination der festgestellten Bedeutung wahmehmen muß. So steht vor ihrem Bewußtsein eine ganz bestimmte Aufgabe, in den dargebotenen Lautkomplexen nicht irgendeine Bedeutung überhaupt, sondern eine bestimmte, in der vorgeführten sinnlosen Zeichnung vorgefundene herauszulesen. Dementsprechend charakterisieren die Versuchspersonen ihre Erlebnisse an diesem Stadium der Versuche wie folgt:

Bu. II. Der eine Teil der Zeichnung sah einem Stock ähnlich, an dem ein Strick befestigt ist. Beim Lesen der Lautkomplexe wandte sich meine Aufmerksamkeit dem Komplex Ketibi zu, der von mir als Kasus instrumentalis von Keti (Stock) aufgefaßt wurde: „ketith, d. h. ,ein Strick mit einem Stock", dachte ich mir, das wäre doch am passendsten für die Benennung der Zeichnung.

Wenn wir auch die anderen analogen Beobachtungen der Versuchspersonen in Betracht ziehen, können wir kurz das Ergebnis folgendermaßen formulieren: Bei der Wahrnehmung der Lautkomplexe wird von 
der Versuchsperson entweder der ganze Komplex oder einer seiner Teile apperzipiert und mit der in der Zeichnung aufgedeckten Bedeutung assimiliert. Dieser Prozeß setzt, wenn nicht die Tatsache des vollendeten Sprechenkönnens, so mindestens einen mehr oder weniger reichen Vorrat bekannter und gewöhnlich anwendbarer Wörter voraus.

Man könnte meinen, daß es regelmäßig so sei, daß die Versuchsperson mit einer bestimmten Vorstellung, die für sie die Bedeutung des zu benennenden Objektes ausmacht, an die Lautkomplexe herantrete und diese in der Richtung zu dieser Vorstellung assimiliere. Das ist aber nicht der Fall: Eine solche eindeutige oder eindimensionale Determination der Wahrnehmung der Lautkomplexe kommt nur in seltenen Fällen zustande. Häufiger (ganz besonders aber bei dem oben charakterisierten, phantasierenden Typus) geht der Aufbau der Bedeutungsvorstellung nach dieser oder jener Richtung dem Durchsehen der Lautkomplexe nicht voran, sondern er folgt demselben nach. Es schwebt in solchen Fällen der Versuchsperson die Bedeutungsvorstellung allgemein und schematisch vor, und sie wird konkret und anschavlich vollendet erst an der Hand der Lautkomplexe, welche durch ihren Klanglaut im Bewußtsein der betreffenden Person irgendeine in das Schema der Bedeutung irgendwie hineinpassende Vorstellung wachrufen.

Wesentlich anders gestaltet sich die Sachlage in denjenigen Fällen, in welchen die Bedeutungsvorstellung des zu benennenden Objektes nicht anschaulich, oder in der Form eines unanschaulichen Gedankens, sondern bloß emotionell oder in der Form eines ,unbestimmten allgemeinen Eindrucks" vergegenwärtigt wird.

Was zunächst den ersten emotionalen Typus anbetrifft, so hat es sich herausgestellt, daß er sich auch bei der Wahrnehmung der Lautkomplexe geltend macht, und zwar in der Weise, daB jeder Lautkomplex mit einer bestimmten Gefühlslage verbunden wird, die dann mit derjenigen des zu benennenden Objektes assimiliert wird.

Tsi. IV. Assudich ist wenig passend, denn es mutet mich nicht angenehm an, ebensowenig nagtachi, denn es wrikt noch weniger angenehm...

Scha. IV. . . Pozero ist mild und der Gefühlslage des Objektes mehr passend, viel mehr als z. B. re $\varphi$ ibu...

Diejenigen Versuchspersonen, die die sinnlose Zeichnung auf sich allgemein wirken lassen und ihre Bedeutung nur in einem undefinierbaren allgemeinen Eindruck sehen, verhalten sich bei der Wahrnehmung der Lautkomplexe in völlig analoger Weise:

Tschi. VI. Deichudsa scheint mir am passendsten zu sein. Es macht auf mich einen solehen Eindruck, daß ich gerade diesen Komplex wählen muß. Er ist eben am passendsten, aber warum, kann ich nicht angeben...

Wie regelmäßig und auf welche Weise dieser allgemeine Eindruck erlebt wird, darüber geben folgende Beispiele Rechenschaft, die ich den speziell für diesen Zweck angestellten Versuchen entnehme: 
Scha. II. q̇šaro. Tch habe dabei einen eigenartigen Eindruck, den ich übrigens gar nicht zu illustrieren vermag. Dasselbe muß ich auch betreffs kosalo sagen. Irgendeine Illustration zu finden, gelingt mir nicht. Aber das eine ist doch klar: Wenn ich von einem zum andern übergehe, so erlebe ich eine Art Kontrast ...

Scha. III. Derselbe Kontrast scheint zwischen decōoci und rephibu zu bestehen. Deழ̣odi macht, den Eindruck etwa eines zurückgebliebenen, wenig entwickelten, passiven Menschen. Reqibu dagegen ist etwas Schwerwiegendes, etwas Weises. Äußerlich ist das ein robuster Mann mit grauem Vollbart; er hat etwas in der Hand, ein Buch...

Cho. I. Lakosu. Das ist etwas, was ganz verschieden ist vom dukasi. Dies ist etwas Schweres, was sich auf den Boden stützt, während jenes etwas Leichtes ist: Es kann in der Luft schweben und es ist außerdem sauberer und heller als dukasi, das mir mehr als etwas Rotes erscheint ...

Es besteht noch ein ganz eigenartiger Weg, den manchmal einige Versuchspersonen bei der Wahrnehmung der Lautkomplexe einschlagen. Wie aus den unten angegebenen Beobachtungen leicht ersichtlich ist, stellen die sinnlosen Lautkomplexe für einige der Versuchsperson ganz bestimmte Gestalten dar, und zwar dermaßen bestimmte, daß die Versuchspersonen nicht selten imstande sind, sie zeichnerisch wiederzugeben.

Tsi. I. q̇ešaru. Es ist sehr ähnlich der zu benennenden Zeichnung. Seine Gestalt ist nicht gerade deutlich, aber sie sieht ungefähr so aus wie ein Storch...

II, III, IV. qetiঠ $i$ trägt die Gestalt der einfachen Linien. Rağešhi ist viel komplizierter.

Tekadi ist durch wellenförmige Linien wiederzugeben...

Techo. IV. Isakud hat eine sehr deutliche Form; ich kann sie sogar nachzeichnen (die Versuchsperson zeichnet dann die ihr vorschwebende Gestalt).

Es kommen also Fälle vor, bei denen ein Lautkomplex, obwohl er keine sprachlich formulierbare anschauliche oder unanschauliche Bedeutungsvorstellung anregt, trotzdem aber einen individuellen ihm eigentümlichen Wert trägt, der sich entweder in einem emotionalen Zustand oder in einem allgemeinen, nicht weiter formulierbaren Eindruck, oder endlich in einer ihm eigentümlichen Gestalt ausdrückt.

\section{c) Die Grundlagen der Namengebung.}

Wie wird der Lautkomplex mit der sinnlosen Zeichnung als ihr Name in Verbindung gebracht? Was ist die Grundlage, auf welcher sich der Akt der Namengebung abspielt?

Wir haben gesehen, da $B$ zwischen der Vorstellung des zu benennenden Objektes und der des als Namen auszuwählenden Lautkomplexes typisch sich ein Mittelglied einschiebt, welches unserer Analyse zufolge als eine Art der Bedeutungsvorstellung des zu benennenden Objektes anzusprechen ist. Es hat sich dazu noch herausgestellt, daß diese Bedeutungsvorstellung ihre individuelle Ausprägung oft erst durch die Einwirkung des ausgewählten Lautkomplexes erhält (s. S. 31). Es ist also klar, daß es eben diese Bedeutungsvorstellung ist, die am Prozeß 
der Namengebung als Vermittlerin mit beteiligt ist, und zwar auf versohiedene Weisen:

1. Am häufigsten wird die Bedeutungsvorstellung durch ein gewöhnlich verwendbares Wort im Bewußtsein repräsentiert, und nun übernimmt dieses die Vermittlungsfunktion zwischen der Zeichnung und dem Lautkomplex. Dieser Weg scheint für den modernen Menschen der gangbarste zu sein; er weist verschiedene Abarten auf:

a) Es wird eine gewöhnlich gebrauchte sprachliche Bezeichnung der Bedeutungsvorstellung festgehalten, und nun wird als passend derjenige Lautkomplex erwählt, der nach seinem Lautgehalt der angegebenen Bezeichnung am nächsten steht:

Bu. I. Die Zeichnung faßte ich als eine Einrichtung zum Sitzen auf. Bei der Auswahl der Lautkomplexe fiel mir de@̣osi als der passendste auf. Entscheidend war da vei die Silbe de, die an das Präfix vom daddoma (georgisch: sitzen) erinnerte ...

b) Oder man begnügt sich auch mit denjenigen Lautkomplexen, die nur einer mit der Bedeutungsvorstellung assoziativ verknüpften anderen Vorstellungen ganz oder teilweise ähnlich klingen:

Cha. II. Die Zeiohnung hat in mir die Vorstellung einer Nase hervorgerufen. Unter dem Lautkomplexen wählte ich qeti $i$, denn die zwei letzten Silben klingen wie Didi (georgisch: Finger), DiDi aber ist auch ein Körperteil...

c) Es gibt auch Fälle, bei denen die Versuchspersonen nicht mit einer schon endgültig ausgewählten Bezeichnung der Bedeutungsvor. stellung an die Lautkomplexe herantritt und in ihnen eineähnlich klingende Komponente sucht, sondern die Versuchsperson ist zufrieden, wenn sie dort irgendeinen Komplex findet, der ähnlich einem die Bedeutungsvorstellung irgendwie charakterisierenden oder ihr zukommenden Worte klingt (vgl. oben S. 32).

Scha. IV. Die Zeichnung hat in mir die Vorstellung eines im psychologischen Institut vorgeführten Bildes erweokt: Eine Maus hinter dem vollen Mehlsack. Beim Durchlesen der Lautkomplexe zog ich utipuk den anderen vor, weil es ähn. lich dem georgischen Wort utipari (frech) klingt, und die Maus hinter dem Sack sah doch frech aus.

Auch die Versuchsperson Me. wählt dasselbe utipok aus, und zwar aus dem. selben Grunde: nur frech scheint ihr die ganze Zeichnung insofern gu sein, als sie in derselben keinen Sinn herauszufinden vermag.

d) Es kam auch vor, daß die Versuchspersonen einen Lautkomplex nicht wegen einer Ähnlichkeit mit einer bestimmten sprachlichen Bezeichnung der Bedeutungsvorstellung wählten, sondern deswegen, weil dieser Lautkomplex im Gegensatz zu den anderen sie „wie ein wirkliches, obgleich auch unbekanntes Wort" anmutet:

Scha. I. Pozero scheint am passendsten: er klingt wie ein italienisches Wort, während die andern ganz sinnlos und absolut nichtssagend sind.

Scha. II. Rašequ scheint am meisten zu passen, denn es macht einen Eindruck, als ob es ein abchasisches Wort wäre. 
Wir sehen also, daß zum charakteristischen Merkmale für diesen ersten, am häufigsten gangbaren Weg der Namengebung die Tatsache der Sprachenexistenz und Sprachenkenntnis dient.

2. Es gibt aber auch andere Prozesse, die dem Akt der Namengebung zugrunde liegen. Wir haben schon oben erwähnt, daß Lautkomplexe ihre eigene Gestaltqualität besitzen, die sehr leicht mit der der sinnlosen Zeichnung verglichen werden kann. Nun ist es begreiflich, daß von den Versuchspersonen nur derjenige Lautkomplex zum Namen ausgewählt wird, dessen Gestalt der der Zeichnung am besten paßt.

Tsi. I. Bei der Betrachtung der Zeichnung fiel mir nichts anßer ihrer Geatalt auf. Unter den Lautkomplexen wählte ich chescharu. Es geschah nämlich so: Zuerst habe ich alle gegebenen Lautkomplexe durchgesehen, indem ich genau auf die „Lautgestalt" derselben achtete. $\dot{q}$ 'šaru zeichnete sich von allen anderen gerade durch seine der Zeichnungsform am besten passende Gestalt ans.

Tsi. III. Die Zeichnung rief in mir die Vorstellung einer Speerspitze hervor; diese aber wurde bald vergessen, so daß sie keinen Einfluß auf die Wahl der Lautkomplexe ausgeübt hat. Bei der Wahl des passenden Namens wurde ich vielmehr durch die allgemeine Form der Zeichnung geleitet: tekhati ist in dieser Beziehung besonders passend für die Zeichnung. pirebu dagegen kann gar nicht in Betracht kommen, denn es ist etwas mehr Wellenförmiges...

Irgendwelche Worthilfen kommen bei dieser Kategorie nicht vor; die Versuchsperson benimmt sich, als ob sie kein einziges Wort kenne, das ihr irgendeine Hilfe gewähren könnte.

3. Von vielen Beispielen der Fälle, wo ein emotionales Moment entscheidend wird, seien nur die folgenden angeführt:

Scha. VI. Anfangs erschien mir die Zeichnung völlig sinnlos; nachträglich tauchte in meinem Bewußtsein die Vorstellung eines Fisches auf. Bei der Wahl der Lautkomplexe schwankte ich zwischen rephibu und pozero. Dieses ist etwas Angenehmes, Lustbringendes, und weil die Vorstellung des Fisches mit der des Meeres verknüpft war, diese aber lustbetont ist, wählte ich schließlich pozero aus.

Tsi. IV. Diese Zeichnung konnte ich mit keiner Vorstellung in Verbindung bringen. Sie hinterlie $B$ in mir ein entschieden angenehmes Gefühl. Bei der Wahl der Lautkomplexe sah ich, daß weder assudiqu noch nagia $\dot{a} i$ paßten. Uberip $\mathrm{da}$ gegen erweckte in mir ein passendes Gefühl. Deshalb habe ich es ausgewählt.

Die Verknüpfung geschieht hier zwischen dem Lautkomplex und der Vorlage an der Hand der emotionalen Gesamtlage, die durch die Wahrnehmung der beiden Glieder hervorgerufen wird. Im Momente des Vergleichens und Beziehens haben wir es nicht entscheidend mit den Vorstellungen der beiden Glieder zu tun, sondern mit durch diese hervorgerufenen Gefühlen.

Da die Wahrnehmung des zu benennenden Objektes und folglich auch das durch sie veranlaßte Gefühl der Wahrnehmung des Lautkomplexes und ihrem Gefühlston vorangeht, so ist es klar, daß das erste Gefühl entweder gedächtnismäßBig, oder aktuell im Bewußtsein der Versuchspersonen verhanden sein und auf diese Weise auf das Gefühl des zweiten Gliedes bezogen werden muB, oder, ist dies nicht der Fall, der Vergleich ohne das erste Glied vollzogen, oder schließlich die Beziehung willkürlich und grundlos erlebt werden muß, ohne daB die Versuchspersonen das Gefühl des Zusammenpassens haben. 
Wir haben spezielle Versuche angestellt, um die letzte Möglichkeit zu prüfen, und es hat sich herausgestellt, daß hier von Willkürlichkeit und Zufälligkeit des Beziehens nicht die Rede sein kann. Das Erlebnis des Passens, folglich auch der Art des Vergleichens war zweifellos da. Also, wir müssen vielleicht annehmen, daB das erste Glied bei dem Akte des Vergleichens im BewuBtsein irgendwie vor * handen war, und zwar entweder gedächtnismäßig oder aktuell. Es ist aber weder das eine noch das andere möglich: das erste nicht; denn das Gefühl, wie darauf schon Külpe mit Recht hingewiesen hat, wird durch die Aktualität charakterisiert und es kann also reproduktiv nicht vorhanden sein. Das zweite auch nicht, $d . h$. es konnte auch aktuell nicht gegenwärtig sein, denn da dieses und zu ihm als passend erlebtes Gefühl offenbar einer und derselben Natur sind, beide miteinander verschmelzen müßten, und es bliebe dann nichts übrig, was zu vergleichen wäre: setzt doch das Vergleichen, wenn nicht den Unterschied, so doch die getrennte Existenz der zu vergleichenden Objekte voraus. Es bleibt also die einzige Mög* lichkeit, und zwar, daß der Beziehungsakt ohne das Vorhandensein des ersten Gliedes vollzogen wird. Ist das aber der Fall, so sind vielleicht daraus in bezug auf die Psychologie der Relation wichtige Schlüsse zu ziehen. Es ist nämlich zuzugeben, da. $\mathrm{B}$ sogenannten tbergangsempfindungen durchaus nicht die Rolle zuzuschreiben ist, welche ihnen als den notwendigen Grundlagen des Relationserlebnisses oft zugesprochen wird.

4. Ich gehe zu der letzten sehr interessanten Gruppe der Angaben der Versuchspersonen über, als deren Beispiel ich folgendes anführen möchte:

Tscho. IV. Ich wollte der Zeichnung irgendeinen Inhalt verleihen. Einen Teil stellte ich mir als Feuerflammen vor; dies hat das Bild der Magier hervorgerufen, die sich ums Feuer versammelt haben, um Prophezeiungen zu machen. Bei der Auswahl der Worte wollte ich mich durch dieses Bild leiten lassen. Beim Durchlesen der Liste aber'sagte ich mir: Nein, hier gibt es nichts Passendes. Trotzdem lenkte ich meine Aufmerksamkeit unwillkürlich auf einen Lautkomplex, und zwar auf evohur, vielleicht deshalb, weil er ähnlich dem armenischen Wort Gott klingt; doch bin ich dadurch gar nicht befriedigt. Es ist sonderbar, daß ich mich in anderen Versuchsreihen ganz anders verhielt: Kaum las ich die Liste der Lautkomplexe durch, so drängte sich irgendein Lautkomplex gleich als vollständig passend auf, und ich fühlte mich gleichsam gezwungen, gerade ihn auszuwählen: Die Vorlage und der Lautkomplex deckten sich vollständig. Hier ist die Sachlage aber wesentlich anders: Evohur bleibt sozusagen für sich und die Vorlage auch für sich, sie decken sich nicht. Die Vorlage macht den Eindruck von etwas Emporschwebendem, während evohur mehr der Erde zu gerichtet ist. Ich erlebe es unangenehm, daß ich nichts Passendes herausfinden kann.

Diese Angabe ist von verschiedenen Gesichtspunkten aus interessant. Ihre allseitige Analyse wäre sehr anziehend. Dennoch wollen wir uns nur auf diejenigen Momente beschränken, die uns speziell für diesen Zusammenhang von besonderer Wichtigkeit zu sein scheinen. Vor allem ist nun hervorzuheben, daß der Versuchsperson der ,Eindruck" von der Vorlage und dem Lautkomplex besonders maßgebend für das Erlèbnis ihres Zusammenpassens zu sein scheint. Der Weg, den die anderen Versuchspersonen am liebsten betreten, um sich die Wahl der Lautkomplexe zu erleichtern, der Weg der bekannten Worthilfen, ist für Tscho dermaßen unzureichend, daß er ihn nicht mal zu betreten versucht; zwar gelingt es ihm, durch ein bekanntes Wort die Verbindung 
zwischen der Vorlage und dem Lautkomplex herzustellen, dies gewährt ihm aber keine Befriedigung: diese Verbindung erscheint ihm zufällig und unbegründet, und obgleich er nichts besseres findet, will er sich doch derselben nicht bedienen. Es ist klar, daß für unsere Versuchspersonen nur die Verbindung als sicher gilt, die sich auf die Gleichartigkeit des Eindrucks der beiden zu verbindenden Glieder gründet.

Es ist außerdem hervorzuheben, daß der Eindruck, den die Versuchsperson von der Vorlage bzw. von dem Lautkomplex hat, nicht als ein allgemeiner, unbestimmter Eindruck zu charakterisieren ist, sondern die Versuchsperson imstande ist, denselben anschaulich auszudrücken, indem sie ihn zu illustrieren versucht: ,es ist etwas Emporschwebendes, während jenes etwas nach unten Gerichtetes ist", sagt Tscho. zur Charakterisierung der Eindrücke, die der Vorlage und dem Komplex zukommen und diese zu verbinden verhindern.

Also können wir zusammenfassend sagen: als Grundlage der Namengebung tritt der Eindruck auf, den die Versuchsperson von beiden zu verbindenden Gliedern bekommt, und den sie durch die passende Illustration zu präzisieren versteht.

Von diesem Typus ist der nächstfolgende zu unterscheiden, obgleich er auch als zu dieser Kategorie gehörend anzusprechen ist:

Bu. III. Bei der Betrachtung der Vorlage suchte ich, aus derselben irgendeinen Sinn herauszulesen. Das gelang mir jedoch nicht. Es blieb der Eindruck von etwas Unbestimmtem, Sinnlosem, Chaotischem. Bei der Auswahl des Lautkomplexes war für mich eben dieser Eindruck maßgebend. Ich wählte den Lautkomplex diqud $a$ aus, der auf mich den analogen Eindruck von etwas Chaotischem machte. Zwar habe ich in beiden Fällen ein ausgesprochenes Unlustgefühl gehabt, aber ich kann bestimmt sagen, daß dieses bei der Wahl des Namens absolut nicht in Betracht kam.

Es ist zweifellos, daß wir hier wesentlich mit einem ähnlichen Prozeß zu tun haben, welcher, wie ihn Versuchsperson Tscho. IV charakteristisch konstatierte: Zur Grundlage des Bewußtseins des gegenseitigen Passens zwischen dem Lautkomplex und der Vorlage tritt in beiden Fällen das Erlebnis der Verwandtschaft der allgemeinen Eindrücke auf, die sich an der Hand der Wahrnehmung der beiden Glieder geltend machen. Der Unterschied ist nur darin zu sehen, daß während in jenem Falle der Eindruck sich durch die Illustrationsvorstellung präzisieren ließ, in diesem Falle nur durch die Eigenschaft des "Chaotischen“ „Sinnlosen" charakterisiert wird. Die Bestimmtheit des Eindrucks von einem solchen Zwischenglied zwischen der Vorlage und dem Lautkomplex nimmt in diesem Falle beträchtlich ab; sie bringt oft nur negative Charakterisierung. Wir haben aber auch Fälle, bei denen diese Bestimmtheit in dem Maße abnimmt, daß die Versuchspersonen keine Mittel zu finden glauben, um das uns interessierende Zwischengliedserlebnis irgendwie verständlich zu machen. 
Us. I. Ich wollte versuchen, mir die Vorlage im allgemeinen einzuprägen, ohne ihr irgendeine Bedeutung zuzuschreiben. Trotzdem wurde ich die Tendenz nicht los, in der Vorlage etwas Bekanntes zu suchen; und so geschah es, daß der Teil rechts apperzipiert und als eine Fahne vorgestellt wurde. Diese Vorstellung leitete mich auch bei der Wahl der Worte. Ich fand aber in der Liste zunächst nichts Passendes. Doch drangen in das Zentrum des Bewußtseins zwei Komplexe ein : $d_{u} \dot{q} i_{\mathfrak{a}}$ und dețosi. Sie schienen mir am passendsten zu sein, nicht weil sie etwa durch ihren Lautbestand an denjenigen des Wortes Fahne (georg. Droša) erinnerten, wie es in den vorhergehenden Versuchen häufig der Fall gewesen ist. Nein, sie machten auf mich einen ganz eigenartigen Eindruck, den ich nicht näher zu charakterisieren vermag, der aber sehr gut zur Vorlage paßte...

Es ist klar, daß die Versuchsperson einen eigenartigen Eindruck hat, der als Grundlage der Namengebung erlebt wird, den sie aber nicht näher zu illustrieren vermag. Andere Versuchspersonen, die auch ein deutliches Erlebnis des Zusammenpassens von Wort und Objekt haben, sagten z. B.: „Der Lautkomplex paßt sehr gut, besser als alle anderen, aber warum, das kann ich absolut nicht angeben'. . . das ist die gewöhnliche Formel, deren sich diese Versuchspersonen in solchen Fällen bedienen.

Das Vorhandensein dieses eigenartigen Zustandes, seine Faktizität, ist durch unsere Versuche sichergestellt. Uber seine funktionale Bedeutung könnte man vieles sagen ${ }^{1}$ ), und es wäre vielleicht sehr lehrreich, auch das sog. Sprachgefühl, das durchaus noch nicht geklärt ist, einmal vom Gesichtspunkte dieses eigenartigen Zustandes aus zu betrachten.

In bezug auf die psychologischen Grundlagen der Namengebung haben wir also auf Grund unserer Versuche zusammenfassend folgendes: zu berichten:

Bei der Verbindung, die zwischen dem zu benennenden Objekt und dem Lautkomplex hergestellt wird, tritt ein ganz bestimmter psychischer Zustand auf, der als Grundlage der Namensgebungsfunktion anzusprechen ist; das Wort tritt also im allgemeinen nicht zufällig an das beliebige Objekt heran, um dann als dessen Name zu funktionieren, sondern es scheinen hier bestimmte gesetzmäßige Faktoren wirksam zu sein. Dies sind: a) Angleichung des Lautbestandes der gegebenen Lautkomplexe an denjenigen des einer der bekannten Sprachen entnommenen Wortes, das zum Ausdruck der apperzipierten Bedeutung des zu benennenden Objektes gebraucht wird (assoziativer Faktor); b) das Erlebnis des Zusammenpassens von erfaßter individueller Gestalt des Lautkomplexes und des Objektes (Gestaltverwandtschaftsfaktor);

1) Einiges habe ich schon allgemein in einem Aufsatz hervorgehoben, der unter dem Titel Impersonalia in einer georginischen Zeitschritt (, Tschaceri Mezriērebac, 1, 1-30. Tiflis 1923) erschienen ist. 
c) das emotionale Zusammenpassen derselben (emotionaler Faktor) und d) das Erleben des eigenartigen Zustandes, der die Wahrnehmung der beiden Komponenten begleitet und von den Versuchspersonen als ein allgemeiner Eindruck bezeichnet wird. Weil dieser Zustand deskriptiv schwer oder gar nicht erfaßt werden kann, wollen wir ihn als psychischen miefenschichtfaktor bezeichnen. Die letzten 3 Falstoren fassen wir ins. gesamt als sachliche Faktoren zusammen.

\section{d) Der Wert der verschiedenen Faktoren.}

Ganz von selbst entsteht die Frage, wie der sprachfundierende Wert der genannten Faktoren anzusetzen ist.

Zweifelsohne muß hier eine sichere Kontrollbedeutung der Frage zugeschrieben werden, wie ,sicher", „fest" die Verbindung zwischen dem Lautkomplex und dem Objekt ist, die auf Grund von diesem oder jenem Faktor erlebt wird. Zufälligerweise hat im Laufe der Hauptversuche eine der Versuchspersonen unaufgefordert eine Wertung der Falztoren selbst vollzogen, indem sie sich in bezug auf den assoziativen Faktor folgendermaßen äußerte:

Tscho. IV. In diesem Falle hat das Wort einen zufäligen Charakter; es taugt eigentlich gar nicht zum Namen des vorgelegten Objektes...

Der assoziative Faktor scheint nicht imstande zu sein, das ,rechte Wort" in ,befriedigender Festigkeit" zu lieferm. Wir haben bei einigen Tersuchspersonen hierzu spezielle Versuche angestellt. Es wurden nach einer genügend langen Pause den betreffenden Versuchspersonen dieselben Zeichnungen vorgelegt und zunächst nach den Namen gefragt, die sie in der Hauptversuchsreihe angegeben hatten (A-Versuch). Wenn den Versuchspersonen das Gedächtnis versagte, wurde ihnen dann die entsprechende Lautkomplexenliste in die Hand gegeben, damit sie den früher gewählten Namen wiederfinden konnten (B-Versuch). Die Argebnisse beim A.Versuch zeigten:

1. Bei Komplexen, die auf Grund des assoziativen Faktors ausgewahlt worden waren, zeigte sich oft ein festeres Haften im Gedächtnis, aber nur hinsichtlich eines Teiles des Lautkomplexes, z. B.:

Me. I. Den ganzen Komplex weiß ich nicht mehr. Ich weiß aber, daß ich mit Hilfe dichwi die Verbindung hergestellt habe; es muß im Komplex d, w, $\dot{\mathrm{q}}$-Laute vorhanden sein...

oder die Grundlage der Angleichung allein war noch im Gedächtnis:

Me. III. Die Vorlage habe ich dem Felsen angeglichen; welchen Komplex ich aber zum Namen gewählt habe, weiß ich nicht mehr.

Beim B-Versuch, beim Wiederaussuchen des schon einmal genannten Lautkomplexes in der Liste, zeigte sich Leitung durch dieselbe Grundlage, deren sich die Versuchsperson in dem Hauptversuch bedient hatte und gut im Gedächtnis behalten war; die Versuchsperson aber, in der Wahl nun sehr häufig dabei irregeführt, sie wählt nun einen anderen Lautkomplex. 
Ne. IV. Als Hilfe habe ich das Wort utiproba (Frechheit) verwendet. Welchen Komplex ich aber damals genannt habe, weiß ich nicht mehr...

Nun wählt die Versuchsperson in der überreichten Lautkomplexenliste tuqoøa, während sie in der Hauptreihe den ganz anderen Komplex utikop gewählt hatte.

2. Wesentlich anders gestaltet sich das Bild in den Fällen, in denen andere Faktoren beim Akte der Namengebung wirksam waren: Im AVersuch versagte das Gedächtnis hier zumeist vollständig: Die Versuchsperson weiß nicht, welchen Komplex sie gewählt hat; sie kann auch nicht einzelne Teillaute angeben, die im gewählten Komplex figurierten. Wenn ihr nun (im B-Versuch) die betreffende Lautkomplexenliste überreicht wurde, so ,,wußte ${ }^{c}$ sie typisch zwar auch nicht, was sie in der Hauptreihe genannt hatte, nichtsdestoweniger wählte sie aber fast in allen Fällen dieselben Komplexe aus.

Man könnte meinen, daß hierbei die Versuchsperson den früher genannten Komplex in der Liste wiedererkenne und nurdeshalb denselben als passenden Namen nenne. Das scheint nicht der Fall zu sein. Hier seien 2 Beispiele angeführt, die in dieser Beziehung wohl sehr instruktiv sind:

Tsi. IV. „Ich weiß nicht, was ich ausgewählt habe. Ich sehe aber jetzt in der Liste zwei Worte (er nennt diese), die fast gleich passend zu sein scheinen. Welches von beiden aber am passendsten ist, kann ich nicht entscheiden ... Doch scheint mir das letzte immerhin besser zu passen." Das war aber gerade der Lautkomplex, den die Versuchsperson auch in der Hauptreihe gewählt hatte.

Oder:

Tsi. I. Ich glaube, ich habe damals hier in der Liste tiðeka ausgewählt. Jetzt aber sehe ich, daß hier ein noch viel passenderer Lautkomplex vorhanden ist, nämlich qंešaru.

Die Versuchsperson aber hatte in der Hauptreihe nicht tiذeka ausgewählt, wie sie selbst glaubt, sondern gerade den Komplex quešaru, der ihr jetzt am passendsten erscheint.

Auch hinsichtlich der Reaktionszeiten scheint ein Unterschied vorhanden. Wir greifen aus unseren Protokollen die Zahlen heraus, die sich auf die Reaktionszeiten von typisch sich unterscheidenden Versuchspersonen beziehen. Dies sind die Versuchspersonen Meund Tsi. : bei jener ist der, ,assoziative“ Faktor am meisten wirksam, bei dieser aber sind gewöhn. lich die übrigen Faktoren tätig. Aus der Gegenüberstellung der Reaktionszeiten der beiden Versuchspersonen ist ersichtlich, daß die Reaktionszeiten der Versuchsperson $M e$, also die Reaktionszeiten der sich nach dem assoziativen Faktor richtenden Versuchsperson, im Durchschnitt etwa 6 mal so groß sind als diejenigen der Versuchsperson Tsi, bei welcher gewöhnlich die übrigen Faktoren wirksam sind. Man könnte gegen diesen Schluß vielleicht mit Recht einwenden, daß der Unterschied der Reaktionszeiten hier nicht aus der Verschiedenheit der Namensgebungsfaktoren, sondern aus derjenigen der individuellen Gleichung entspringen. Wenn wir aber die Zeiten der beiden Versuchspersonen noch einmal einzeln durchsehen, so finden wir, daß die Versuchsperson 
Me die minimale Reaktionszeit beim Versuch III, die Versuchsperson Tsi aber die maximale Reaktionszeit beim Versuch I und II aufweist. Sehen wir die Protokolle durch, so finden wir, daß bei der Versuchsperson Me gerade beim Versuch III der assoziative Faktor versagt hat und der emotionale Faktor aufgetreten ist, während bei der Versuchsperson $T s i$ gerade in der Versuchsreihe I und II eben der assoziative Faktor zur Wirksamkeit gekommen ist.

Nach all diesen Befunden scheint der sprachfundierende Wert der sachlichen Faktoren viel höher als der des assoziativen Faktors. Spontane Äußerungen der Versuchspersonen, die wiederholte, nicht auf Gedächtnishilfen ruhende Auswahl derselben Komplexe, die auf Grund der sachlichen Faktoren einmal als passende erlebt wurden, die verhältnismäßig geringe Dauer der Reaktionszeiten, das alles weist auf den vergleichsweise hohen sprachfundierenden Wert hin, den die sachlichen Faktoren $z u$ besitzen scheinen.

Ist aber der Wert der sachlichen Faktoren wirklich so hoch einzuschätzen, so ist es konsequenterweise zu erwarten, daß die Lautkomplexe bei ein und derselben Person ein und demselben Objekt konstant zugeordnet werden und daß in bezug auf diese Zuordnung eine weitgehende Utbereinstimmung unter den verschiedenen Versuchspersonen besteht.

Um diesen Schluß auf seine faktische Richtigkeit hin zu prüfen, müssen wir unsere Versuchsergebnisse einer quantitativen Bearbeitung unterziehen. Allerdings ist unser Material für die quantitative Begründung der allgemeinen Ergebnisse nicht ausreichend; dennoch ist es nicht ohne Interesse, die Resultate daraufhin zu betrachten. Zunächst die Frage: ob alle dargebotenen Lautkomplexe mit gleicher oder verschiedener Häufigkeit als Namen zur Geltung kommen?

Im ganzen wurden $\mathbf{4 2}$ verschiedene Lautkomplexe gegeben, die nach der Zahl der Versuchspersonen 9- bzw. 8 mal zur Erscheinung kamen, d. h. jeder Komplex hat während der ganzen Versuchsserie 9. bzw. 8 mal die Gelegenheit gehabt, ausgewählt zu werden. Trotzdem wurden als Namen der ausgewählten Objekte im ganzen 29 verschiedene Komplexe, d. h. $68 \%$ genannt; die übrigen $32 \%$ kamen bei den vorgelegten Objekten überhaupt nicht in den Wahlresultaten vor.

Es kann sich hier wohl nicht um einen Zufall handeln: $32 \%$ ist nicht die Zahl, die aus der Zufälligkeit zu erwarten wäre.

Wie sind nun die Zahlen der Zuordnung der Lautkomplexe zu den Objekten? Die nebenstehende Tabelle gibt darüber Aufschluß.

\begin{tabular}{r|c}
\hline Vorlage & $\begin{array}{c}\text { Ubereinstimmung der } \\
\text { Versuchsperson in \% }\end{array}$ \\
\hline I & 45 \\
II & 40 \\
III & 25 \\
IV & 40 \\
V & 38 \\
VI & 40
\end{tabular}


Wie aus der Tabelle klar ersichtlich ist, haben $45 \%$ von sämtlichen Veršuchspersonen für die erste Figur einen und denselben Lautkomplex am besten passend bezeichnet; die übrigen $55 \%$ sind in der Bezeichnung der Vorlage nicht einig. Ähnlich, etwas geringer, ist die Ubereinstimmung bei II-VI. Für die zweite Figur sind von allen Versuchspersonen überhaupt nur 3 verschiedene Lautgebilde genannt worden, ebenso für die 4. und 6. Figur. Die 3. und 5. Figur ergab die Ubereinstimmung von $25 \%$ bzw. $38 \%$.

Bei der zufälligen Herstellung und ziemlich großen Zahl der auszuwählenden Lautkomplexe ist also der Prozentsatz der Übereinstimmung der Versuchspersonen beinahe unerwartet groß ausgefallen.

Nun fragt es sich, welche der genannten Faktoren dieser Konstanz der Zuordnung zugrunde liegen. Hier ergibt sich nun charakteristisch, daß der assoziative Faktor nur in einem einzigen Fall die Utbereinstimmung der Figurenbenennung ergeben hat; die anderen Fälle sind fast gleichmäßig unter den sachlichen Faktoren verteilt.

Der assoziative Faktor scheint eigentlich nur als ein Ersatzfaktor zu fungieren, auf welchen der sprechende Mensch meist geneigt ist, zurückzugreifen.

Die übrigen von uns aufgedeckten Faktoren scheinen die entscheidenden zu sein. Diese Faktoren sind es wohl, die die sprachliche Zuordnung begründen und also dem Ganzen des ,Wortes": der Verschmelzung „Lautkomplex-Bedeutung“" zugrunde liegen. Das Wort würde nicht zufällig assoziations- und blinderfahrungsmäßig an das beliebige Objekt herantreten und auf diese Weise als Glied zufälliger Verkettung funktionieren, sondern es scheinen bestimmte sachliche Gesetzmäßigkeiten entscheidend, denen die menschliche Seele auch auf dem Gehiete der Namengebung folgt.

Was die Wortentstehung anbetrifft, sind in den Ansichten der Sprachforscher bzw. der Sprachphilosophen von alters her zwei grundentgegengesetzte Richtungen zutage getreten. Der Subjektivismus einerseits will gerade das Subjekt als $\mathrm{Maß}$ aller Dinge gelten lassen. Die assoziative Einstellung bei der Erklärung der psychologischen Erscheinungen ist ihm von Hause aus eigentümlich; and so kommt es, daß schon der Sophistik die Sprache ganz im Kreise des subjektiven Vorstellens eingeschlossen erscheint: die Beziehung der Lautgebilde zu den bezeichneten Vorstellungen ist für sie zufällig, sie hat absolut keinen Grund im objektiven Sachverhalt.

Der Objektivismus dagegen sucht den Kreis der subjektiven Vorstellungssphäre zu zersprengen und den Grund der Verbindung in der objektiven Sphäre nachzuweisen. Curtius hat einmal den Sinn des Objektivismus prägnant zum Ausdruck gebracht, indem er sagte: ,Man hat die Bebauptung, daß die ältesten Wörter irgendeine Beziehung der Laute zu der bezeichneten Vorstellung voraussetzen, oft verlacht und verspottet. Dennoch ist es schwer, ohne diese Annahme die Entstehung der Sprache zu erklären. Auf 
jeden Fall wohnt auch in den Wörtern weit vorgeschrittener Perioden die Vorstellung wie eine ,Seele" ${ }^{\text {" }}$ ).

Cassirer führt uns noch unmittelbarer in das Zentrum des Problems des Objektivismus, indem er den Schwerpunkt desselben ganz präzis zur Frage zuspitzt, ob es ,,eine innere objektive Wahrheit der Benennungen selbst gibt" ${ }^{\text {"2 }}$ ).

Es ist klar, daB die Ergebnisse unserer Versuche ganz unmittelbar in der Richtung der Gedankengänge des Objektivismus liegen. Wie aber die Tatsachen im Näheren liegen, müssen weitere Untersuchungen zeigen; manche Probleme sind hier wichtig, z. B.: Onomatopöie im $\operatorname{Sinn}$ bloß gleicher Einzelteile scheint nicht wesentlich mitzuspielen. Zur Erforschung dieser - und ähnlicher - Fragen sind weitere Untersuchungen unumgänglich.

1) G. Curtius, Grundzüge der griechischen Etymologie $\mathbf{3}, 96$ (zitiert nach Cassirer, Philosophie der symbolischen Formen S. 139).

2) E. Cassirer, a. a. 0. 1, 133.

(Eingegangen am 15. November 1923.) 